\title{
IMPACTO DE LA PESCA RECREATIVA SUBMARINA SOBRE LAS POBLACIONES DE SPARISOMA CRETENSE (LINNAEUS, 1758) EN LAS AGUAS INTERIORES DE LA ISLA DE TENERIFE (ISLAS CANARIAS)
}

\author{
Javier Goikoetxea*, Alfredo Fernández, \\ Arnau Andreu y Pablo Martín \\ Universidad de La Laguna
}

\section{RESUMEN}

Dentro de la pesca recreativa, la pesca submarina en Tenerife supone un colectivo inferior en comparación con el resto de modalidades, aunque su rendimiento en términos de biomasa extraída por pescador y hora es superior. La realización de este estudio surgió debido a la escasez de datos acerca del impacto de la pesca submarina en las costas tinerfeñas. Tomando como referencia a la especie Sparisoma cretense por ser el principal objetivo de esta pesca, se llevaron a cabo muestreos de talla y abundancia en tres zonas de aguas interiores delimitadas para la pesca submarina, situadas tanto en la vertiente norte como en la vertiente sur de la isla, y fueron comparadas con zonas en las que no estaba permitida. Los resultados no muestran diferencias significativas entre estas zonas. Sin embargo, si se observan diferencias en cuanto a la abundancia de individuos de tallas medianas y grandes en función de las distintas localidades, lo que puede estar asociado a distintos factores cuyo análisis sería interesante para futuros estudios.

Palabras Clave: Sparisoma cretense, pesca submarina, zonas de pesca, Tenerife.

\section{IMPACT OF RECREATIONAL SPEARFISHING ON THE SPARISOMA CRETENSE (LINNAEUS, 1758) POPULATIONS IN THE INTERIOR WATERS OF TENERIFE ISLAND (CANARY ISLANDS)}

\begin{abstract}
In recreational fishing, the spearfishing involves a smaller collective than the rest of modalities, although, its yield, in terms of biomass, extracted by fisherman and hour is higher than any other modalities. This study was performed due to the scarcity of data about the impact of underwater fishing in the coasts of Tenerife. Due of being it the main objective of the spearfishing, Sparisoma cretense sampling for its size and abundance was carried out in three interior water areas, situated both in the north and in the south of the island, where the spearfishing is allowed, and then compared with another three zones where it is forbidden. The results show no significant differences between those zones. However, there are significant differences in the abundance between the medium and the big size individuals in the different locations, that might be related with a variety of factors whose analysis may turn of interest for future studies.
\end{abstract}

KeYwords: Sparisoma cretense, spearfishing, fishing zones, Tenerife. 


\section{INTRODUCCIÓN}

La pesca recreativa es una actividad con un gran número de aficionados en la isla de Tenerife, registrándose 33128 licencias en marzo de 2010 y estimándose el número de pescadores recreativos sin licencia entre un 10 y un $20 \%$, suponiendo un $4.2 \%$ de la población insular.

La pesca submarina cuenta con un número de pescadores muy inferior comparado con otras modalidades, 3111 pescadores submarinos frente a los 33128 pescadores recreativos totales. Aunque no existen estudios que constaten el porcentaje de personas dedicadas a esta actividad de manera furtiva (ejercer sin licencia o ejercer con ella pero incumpliendo normativa), debido a lo complicado que es el seguimiento de una actividad ilegal, la experiencia de campo nos da a entender que la existencia de este furtivismo es altamente probable. Además, el estudio de los efectos de la pesca submarina resulta de interés debido a que se extraen $542 \mathrm{~g}$ de pescado por hora frente a los $341 \mathrm{~g} / \mathrm{h}$ y $105 \mathrm{~g} / \mathrm{h}$ extraídos mediante la pesca recreativa con caña desde embarcación y orilla respectivamente (Pascual Fernández et al. 2012).

Una de las especies de mayor interés en la pesca recreativa en Tenerife es la conocida localmente como la vieja (Sparisoma cretense, Linnaeus, 1758), contribuyendo con un $10 \%$ a la biomasa total extraída y pudiendo ser este porcentaje mayor en el caso de la pesca submarina, ya que la boga (Boops boops, Linnaeus, 1758), la lubina (Dicentrarchus labrax, Linnaeus, 1758), la salema (Sarpa salpa, Linnaeus, 1758) y el sargo (Diplodus sp., Linnaeus, 1758) se pescan casi en su totalidad en pesca con caña, contribuyendo en un $28 \%$ al total de biomasa extraída por los pescadores recreativos (Pascual Fernández et al. 2012).

$S$. cretense es una especie demersal de la familia Scaridae, que habita fondos rocosos desde el Mediterráneo oriental con su límite de distribución sur en Cabo Verde (Aquamaps 2016; Reiner 1996). Este pez loro se alimenta fundamentalmente de algas y pequeños invertebrados (Quignard y Pras 1986; Abecasis et al. 2005), y cuenta con un máximo reproductivo entre los meses de julio y septiembre (Abecasis et al. 2005; González 1990). Machos y hembras de S. cretense alcanzan la madurez sexual con tallas y edades similares (De Mitcheson y Liu 2008), siendo estas de $15.5 \mathrm{~cm}$ o a partir de los dos años (Petrakis y Papaconstantinou 1990).

Tenerife consta de 9 zonas delimitadas, donde la regulación de la ley permite la pesca submarina recreativa (BOE-A-1988-4622; BOC 2007/222). De estas 9 zonas, solo 4 están catalogadas como zonas de pesca submarina recreativa de aguas interiores, es decir, en estas puede llevarse a cabo esta actividad dentro de 12 millas de distancia respecto a la costa (fig. 1). Siendo así, puede practicarse la pesca submarina dentro de estas 4 extensiones: T1 (Santo Domingo-Las Aguas), T2 (Punta del Sol-Punta del Viento), T3 (Roques de Antequera - Punta los Organos) y T4 (Boca Cangrejo-Punta del Morro).

* Master en Biología Marina: Biodiversidad y Conservación. Facultad de Ciencias (Biología). Autor para correspondencia: goikoaster@gmail.com. 
El objetivo de este estudio es comprobar si existen diferencias causadas por la pesca submarina, con salida desde costa, en la abundancia y la talla de la vieja (S. cretense) entre las zonas establecidas para este tipo de pesca en aguas interiores y las zonas adyacentes en la que esta actividad no está permitida.

\section{MATERIAL Y MÉTODOS}

\section{Muestreo y ReColección DE DATOS}

Para la realización de este estudio se escogieron tres zonas de pesca en aguas interiores, T2 en la vertiente norte, T3 y T4 en la vertiente sur. Dentro de cada zona se escogió una localidad en la que realizar el muestreo, teniendo en cuenta que el ecosistema tenía que presentar un fondo mayoritariamente rocoso debido a la afinidad de $S$. cretense por este tipo de hábitat. A su vez, se seleccionaron tres zonas control para cada punto de muestreo respectivamente en las que la pesca submarina no estuviese permitida, por estar situadas fuera de los límites establecidos, pero a pocos kilómetros de las localidades de muestreo seleccionadas; evitando de esta forma posibles factores de confusión en los resultados, producidos por variaciones en la zona de muestreo.

Los muestreos se llevaron a cabo en las siguientes playas (figs. 2 y 3):

- Pesca en aguas interiores (T2): Playa del Pris $\left(28^{\circ} 30^{\prime} 47.3^{\prime \prime} \mathrm{N} 16^{\circ} 25^{\prime} 11.7^{\prime \prime} \mathrm{W}\right)$

Zona de pesca prohibida: El Roquillo (28 $\left.32^{\prime} 05.0^{\prime \prime} \mathrm{N} 16^{\circ} 23^{\prime} 52.8^{\prime \prime} \mathrm{W}\right)$

- Pesca en aguas interiores (T3): Igueste de San Andrés (2831'23.7”N 1609'05.9”W)

Zona de pesca prohibida: Añaza $\left(28^{\circ} 25^{\prime} 12.8^{\prime \prime} \mathrm{N} 16^{\circ} 17^{\prime} 44.5^{\prime \prime} \mathrm{W}\right)$

- Pesca en aguas interiores (T4): Boca Cangrejo (28 $\left.24^{\prime} 24.0^{\prime \prime} \mathrm{N} 16^{\circ} 18^{\prime} 50.0^{\prime \prime} \mathrm{W}\right)$

Zona de pesca prohibida: Las Caletillas (28 22'07.3”N 16 $\left.21^{\circ} 40.0^{\prime \prime} \mathrm{W}\right)$

El método llevado a cabo para el muestreo de la abundancia y el tamaño de las poblaciones de $S$. cretense se basó en una toma de datos por tiempo. Los muestreadores por parejas nadaban en direcciones opuestas o divididos entre interior y exterior de la bahía, en función de la climatología y topografía de la zona, durante 25 minutos en paralelo a la línea de costa con una banda de toma de datos de $180^{\circ}$.

Para la recogida de los datos se emplearon tablillas sumergibles, previamente preparadas para clasificar a los individuos observados según sus tallas, estableciéndose los siguientes intervalos: juveniles $(<2 \mathrm{~cm})$, pequeños $(2-10 \mathrm{~cm})$, medianos $(10-20 \mathrm{~cm})$ y grandes $(>20 \mathrm{~cm})$. Esta clasificación se basó en el límite de talla según la regulación para la pesca de esta especie, establecida en $20 \mathrm{~cm}$ (Decreto 155/1986, de 9 de octubre, por el que se establecen las tallas mínimas de captura de peces en aguas interiores del Archipiélago Canario (BOC-1986-1079)). En la normativa nacional, la talla mínima para la pesca de esta especie está establecida en $22 \mathrm{~cm}$ (BOE-A-2015-12897), pero dado que este estudio tiene lugar en las aguas interiores canarias, se decidió acotar la talla mínima basándose en la normativa autonómica, debido a la dificultad, tanto por parte de los practicantes de pesca 
submarina como de los muestreadores; para determinar el tamaño de los individuos con precisión en campo.

Los muestreos fueron realizados durante los meses de febrero y junio de 2019, cubriéndose las estaciones de invierno y primavera respectivamente.

\section{AnÁlisis estadístico}

Para el análisis estadístico se utilizó una base de datos con el software Excel (Microsoft Office) y análisis con el paquete estadístico PRIMER 6 Versión 6.1.11 \& PERMANOVA + Versión 1.0.1. Se realizaron análisis de la varianza por permutaciones (Anderson 2001) en función de la regulación de la pesca submarina y la localidad. Por lo tanto, los modelos incorporaron los siguientes factores: (1) 'Regulación de la pesca' (factor fijo ortogonal con dos niveles correspondientes a pesca submarina permitida y pesca submarina prohibida) y (2) 'Localidad' (factor ortogonal anidado con seis niveles: Boca Cangrejo, Las Caletillas, El Roquillo, Playa del Pris, Igueste de San Andrés y Añaza). Se analizaron las variables: (1) 'Pequeños por unidad de tiempo', (2) 'Medianos por unidad de tiempo' y (3) 'Grandes por unidad de tiempo'. La variable 'Juveniles por unidad de tiempo' no fue analizada ya que durante los muestreos no fueron observados individuos de estas características $(<2 \mathrm{~cm})$, de modo que se consideró prescindir de ella por la ausencia de datos que pudieran aportar interés al estudio. Los resultados significativos $(\alpha=0.05)$ se representaron gráficamente utilizando el software Excel.

\section{RESULTADOS}

En la tabla 1 se presentan los resultados obtenidos tras aplicar un análisis por permutaciones a los datos tomados durante los muestreos. Se observa que no existen diferencias significativas entre las zonas donde la pesca está permitida y las que no están permitidas. Además, en cuanto a los ejemplares con tallas pequeñas $(2-10 \mathrm{~cm})$ y grandes $(>20 \mathrm{~cm})$, tampoco se observaron diferencias significativas en relación a la localidad de muestreo. Por otra parte, en tallas medianas $(10-20 \mathrm{~cm})$, sí se observaron diferencias significativas entre las distintas localidades, destacando El Roquillo e Igueste de San Andrés en tallas medianas (fig. 4). Esta última localidad se encuentra dentro de la zona T3, presentando restricciones de pesca en cuanto a los días semanales permitidos, pudiendo afectar considerablemente sobre la abundancia observada. No se presentan los resultados de juveniles ya que no se encontró ninguno durante la realización del muestreo, presumiblemente por realizarse fuera del pico reproductivo (González 1990). 


\begin{tabular}{|c|c|c|c|c|c|c|c|}
\hline \multicolumn{8}{|c|}{$\begin{array}{c}\text { TABLA 1. PERMANOVA COMPARATIVO DE INDIVIDUOS AVISTADOS POR MINUTO } \\
\text { EN BASE AL TAMANO, ANIDADO EN LA LOCALIDAD Y TENIENDO EN CUENTA } \\
\text { LA PERMISIVIDAD DE LA PESCA }\end{array}$} \\
\hline \multicolumn{8}{|c|}{ PERMANOVA table of results } \\
\hline \multicolumn{8}{|c|}{ Pequeños } \\
\hline Source & $\mathrm{df}$ & SS & MS & Pseudo-F & $\mathrm{P}($ PERM $)$ & UNIQUE PERMS & $\mathrm{P}(\mathrm{MC})$ \\
\hline $\mathrm{Pe}$ & 1 & 547,89 & 547,89 & 0,27 & 0,802 & 10 & 0,79 \\
\hline $\mathrm{Lo}(\mathrm{Pe})$ & 4 & 8098,3 & 2024,6 & 2,05 & 0,079 & 9949 & 0,08 \\
\hline Res & 18 & 17734 & 985,24 & & & & \\
\hline Total & 23 & 26380 & & & & & \\
\hline \multicolumn{8}{|c|}{ Medianos } \\
\hline Source & $\mathrm{df}$ & SS & MS & Pseudo-F & $\mathrm{P}($ PERM $)$ & UNIQUE PERMS & $\mathrm{P}(\mathrm{MC})$ \\
\hline $\mathrm{Pe}$ & 1 & 1593 & 1593 & 0,34 & 0,901 & 10 & 0,76 \\
\hline $\mathrm{Lo}(\mathrm{Pe})$ & 4 & 18544 & 4635 & 4,47 & 0,001 & 9928 & 0,0007 \\
\hline Res & 18 & 18660 & 1036,7 & & & & \\
\hline Total & 23 & 38797 & & & & & \\
\hline \multicolumn{8}{|l|}{ Grandes } \\
\hline Source & $\mathrm{df}$ & SS & MS & Pseudo-F & $\mathrm{P}($ PERM $)$ & UNIQUE PERMS & $\mathrm{P}(\mathrm{MC})$ \\
\hline $\mathrm{Pe}$ & 1 & 2757,5 & 2757,5 & 1,18 & 0,402 & 10 & 0,34 \\
\hline $\mathrm{Lo}(\mathrm{Pe})$ & 4 & 9323,8 & 2331 & 1,31 & 0,212 & 9930 & 0.23 \\
\hline Res & 6 & 0,14 & $2,42 \mathrm{E}-02$ & & & & \\
\hline Total & 11 & 0,74 & & & & & \\
\hline
\end{tabular}

\section{DISCUSIÓN}

En contra de lo esperado con el establecimiento de las regulaciones sobre la pesca submarina en la isla de Tenerife, no se han encontrado diferencias significativas entre las zonas con pesca submarina permitida y aquellas en que se encuentra prohibida su práctica (tabla 1), algo que concuerda con las observaciones del catedrático José Juan Castro, de la Universidad de Las Palmas de Gran Canaria (González 2019).

Es posible que la pesca submarina no cause un impacto, al menos significativo, sobre las tallas de $S$. cretense, siendo un dato que debería corroborarse con un estudio a largo plazo. No obstante, los resultados obtenidos también podrían deberse a varios factores: en primer lugar, no se ejerce la vigilancia necesaria para el control de la pesca recreativa, lo que podría incentivar el furtivismo y no cumplimiento de las regulaciones, declarando entre 43\%-57.9\% de los pescadores no haber sido inspeccionados nunca por ninguna autoridad y siendo aún mayor el porcentaje para inspecciones consecutivas (Pascual Fernández et al. 2012). Además, las bajas sanciones para este tipo de actividad furtiva en aguas interiores, entre $60 \mathrm{y}$ 
$300 €$ (Ley 17/2003, de 10 de abril, de Pesca de Canarias), sumado a la baja probabilidad de ser inspeccionado, podrían no suponer un impedimento para los pescadores furtivos dado el valor de mercado de las capturas, alrededor de $10 € / \mathrm{kg}$. Por otro lado, un sesgo importante en el estudio realizado es obviar los otros tipos de pesca recreativa realizados en la Isla, siendo la pesca con cańa la principal competidora de la submarina y ejerciéndose esta incluso en las zonas donde no está permitida su práctica, por ejemplo, la playa de Las Caletillas, por lo que es posible que esta actividad influyera de forma directa en la abundancia e incluso en la talla de $S$. cretense durante el muestreo. Por último, los pescadores declaran realizar un mayor esfuerzo durante los meses estivales (Pascual Fernández et al. 2012), lo que coincide con la época de desove de S. cretense (González 1990) y podría influir sobre la abundancia de esta especie.

Sin embargo, sí se han encontrado diferencias significativas entre localidades, destacando una mayor abundancia de especies medianas en Igueste de San Andrés y El Roquillo (fig. 4). Esto podría deberse a que estas zonas presentan un hábitat más adecuado para el desarrollo de $S$. cretense, incluyendo fondos rocosos y bosques de macroalgas (Quignard y Pras 1986; Abecasis et al. 2005; González 1990). Además, El Roquillo, que es la localidad con mayor abundancia en todas las tallas, coincide con ser el punto con menor presión antrópica debido a su difícil acceso, por lo que podría existir una correlación entre ambos factores, algo que apoyan las zonas 'no take' de reservas marinas como en la Reserva marina de la Isla Graciosa e Islotes del Norte de Lanzarote (Brito Hernández et al. 2006; Tuya et al. 2004). En cuanto a Igueste de San Andrés, es necesario destacar que es una localidad incluida en la zona T3, por lo que presenta un esfuerzo pesquero supuestamente menor que el resto de localidades debido a las restricciones establecidas en esta zona (fig. 1), factor que podría influir en la abundancia de individuos de tallas medianas (fig. 4).

Aunque no existan diferencias significativas entre las zonas de pesca y no pesca, sería conveniente ampliar los puntos muestreados tanto dentro como fuera de las zonas, con el fin de obtener datos más amplios y precisos. Además, convendría estudiar la variación temporal y estacional de la especie en los lugares muestreados para disminuir el error asociado. A su vez, un estudio poblacional ayudaría a comprender mejor la ecología de esta especie en las distintas zonas estudiadas, favoreciendo una mejor gestión de las aguas interiores de la Isla de Tenerife con pesca submarina permitida y promoviendo el desarrollo de un sistema de control eficaz para esta modalidad de pesca.

\section{AGRADECIMIENTOS}

Queremos agradecer al doctor José Carlos Hernández por la ayuda y tutorización prestada a la hora de realizar este trabajo. 


\section{CONTRIBUCIÓN DE LOS AUTORES}

Conceptualización: JG

Metodología y trabajo en campo: AA, AF, JG, PM

Análisis de datos: AA, AF, JG, PM con ayuda del profesor José Carlos Hernández

Preparación del escrito original: AA, AF, JG, PM

Corrección y edición del escrito definitivo: AF, JG

ReCiBido: septiembre de 2019; ACEPTADO: febrero de 2020 


\section{BIBLIOGRAFÍA}

Abecasis, D., Bentes, L., Ribeiro, J., Machado, D., Oliveira, F., Veiga, P., Gonçalves, J.M.S. y Erzini, K. 2005. First record of the Mediterranean parrotfish, Sparisoma cretense in Ria Formosa (south Portugal). JMBA2-Biodiversity Records Published online

Anderson, M.J. 2001. Permutation tests for univariate or multivariate analysis of variance and regression. Can. J. Fish. Aquat. Sci. 58: 626-639

Aquamaps. 2016. Computer generated distribution maps for Sparisoma cretense (Parrotfish), with modelled year 2100 native range map based on IPCC A2 emissions scenario. Disponible en https://www.aquamaps.org/receive.php?type_of_map=regular (Accedido el 7 de marzo de 2019)

Brito Hernández, A., Barquín Díez, J., Falcón Toledo, J. M., González Lorenzo, J.G., Clemente Martín, S., Hernández, J.C., Toledo Guedes, K., Sangil Hernández, C.A. y Martín García, L. 2006. «Valoración «in situ» de las poblaciones de especies indicadoras del efecto reserva en la Reserva Marina de La Graciosa y los Islotes del Norte de Lanzarote». La Laguna: Viceconsejería de Pesca del Gobierno de Canarias, Universidad de la Laguna-Fundación Empresa Universidad de La Laguna.

De Mitcheson, Y.S. y Liu, M. (2008), Functional hermaphroditism in teleosts. Fish and Fisheries, 9: 1-43. doi: 10.1111/j.1467-2979.2007.00266.x.

Decreto 155/1986, de 9 de octubre, por el que se establecen las tallas mínimas de captura de peces en aguas interiores del Archipiélago Canario. Boletín Oficial de Canarias n. ${ }^{\circ} 125,17$ de octubre de 1986, pp. 2920-2922. http://www.gobiernodecanarias.org/boc/1986/125/boc1986-125-003.pdf.

González, E. 2019. Náutica Canaria. RTVC. Las Palmas de Gran Canaria. Disponible en https:// www.youtube.com/watch?v=6KhMnSzZXhM\&feature=youtu.be (accedido el 12 de marzo de 2019).

González, J.A., 1990. Biología y Pesquería de la Vieja (Sparisoma cretense) en Canarias. Tesis doctoral, Universidad de La Laguna, Tenerife.

Ley 17/2003, de 10 de abril, de Pesca de Canarias. Boletín Oficial de Canarias, n. ${ }^{\circ}$ 77, 23 de abril de 2003, pp. 6123-6145. http://www.gobiernodecanarias.org/boc/2003/077/002.html.

ORDEN de 22 de febrero de 1988 por la que se establecen zonas acotadas en aguas exteriores del archipiélago canario donde se permite la práctica de la pesca deportiva submarina. Boletín Oficial del Estado No 47, 24 de febrero de 1988, pp. 5869- 5870. http://www.boe.es/diario_ boe/txt.php?id=BOE-A-1988-4622.

ORDEN de 29 de octubre de 2007, por la que se acotan, en las aguas interiores de Canarias, las zonas para el ejercicio de la pesca marítima de recreo submarina. Boletín Oficial de Canarias n. ${ }^{\circ} 222,6$ de noviembre de 2007, pp. 25173-25175. http://www.gobiernodecanarias.org/ boc/2007/222/001.html.

Pascual Fernández, J.J., Chinea Mederos, I., Santana Talavera, A., Sosa Rodríguez, P.M., Rodríguez Darias, A.J. y Moreira Gregori, P.E. 2012. La pesca recreativa en Tenerife y su regulación.Tenerife: Cabildo de Tenerife.

Petrakis, G. y Papaconstantinou, C. 1990. Biology of Sparisoma cretense in the Dodecanese (Greece). J. Appl. Ichthyol. 6:14-23. 
Quignard, J.-P. y Pras, A. 1986. Scaridae. pp. 943-944. In P.J.P. Whitehead, M.-L. Bauchot, J.-C. Hureau, J. Nielsen and E. Tortonese (eds.) Fishes of the north-eastern Atlantic and the Mediterranean. UNESCO, Paris, vol. 2.

Real Decreto 1076/2015, de 27 de noviembre, por el que se deroga el Real Decreto 2200/1986, de 19 de septiembre, de regulación de artes y modalidades de pesca en aguas del caladero canario, y se modifica el Real Decreto 560/1995, de 7 de abril, por el que se establece las tallas mínimas de determinadas especies pesqueras, en relación a determinadas tallas mínimas autorizadas para el caladero de Canarias. Boletín Oficial del Estado No 285, 28 de noviembre de 2015, pp. 112544-112545. https://www.boe.es/eli/es/rd/2015/11/27/1076. https://www.boe.es/eli/es/rd/2015/11/27/1076/dof/spa/pdf.

Reiner, F., 1996. Catálogo dos peixes do arquipélago de Cabo Verde. Publ. Avuls. Inst. Port. Invest. Mar. 2:339 p.

Tuya, F., Boyra, A., Sanchez-Jerez, P., Barbera, C.J., Haroun, R., 2004. Relationships between rocky-reef fish assemblages, the sea urchin Diadema antillarum and macroalgae throughout the Canarian Archipelago. Vol. 278: 157-169. 


\section{FIGURAS}

\section{TENERIFE}

aguas exteriores

aguas interiores

Solo permitido sabados, domingos y festivos.

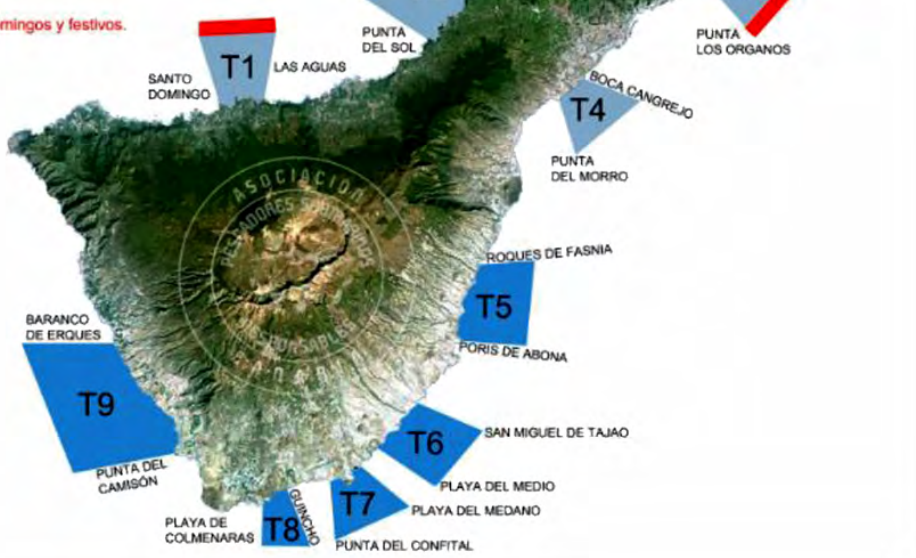

Fig. 1. Mapa de la isla de Tenerife dónde se muestran las zonas habilitadas para las distintas modalidades de pesca recreativa. Fuente http://asociacioncanariapescadoressubmarinos responsables.com/zonas-de-pesca-submarina-en-canarias/.

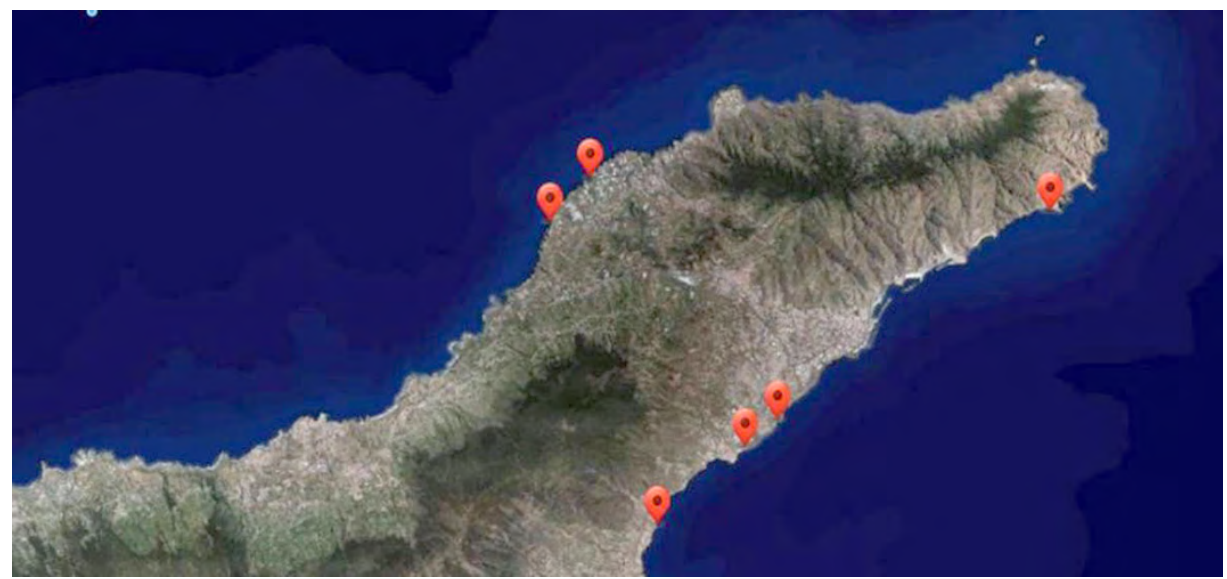

Fig. 2. Visión de satélite del noroeste de Tenerife con las zonas muestreadas. (A: El Pris. B: El Roquillo. C: Igueste. D: Ańaza. E: Bocacangrejo. F: Las Caletillas). Imágenes obtenidas mediante el visor Grafcan. 

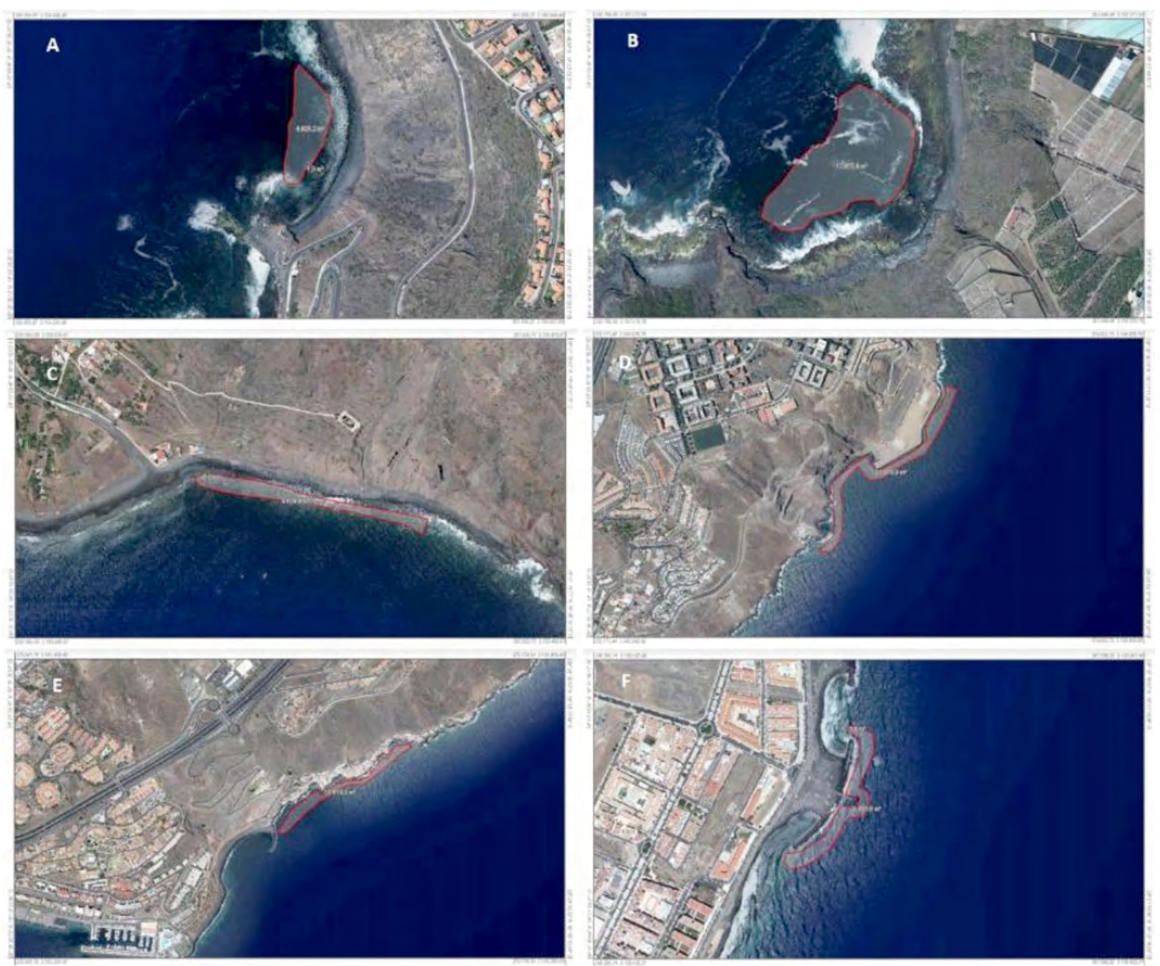

Fig. 3. Visión de satélite de las zonas muestreadas. (A: El Pris. B: El Roquillo. C: Igueste. D: Añaza. E: Bocacangrejo. F: Las Caletillas). Imágenes obtenidas mediante el visor Grafcan.

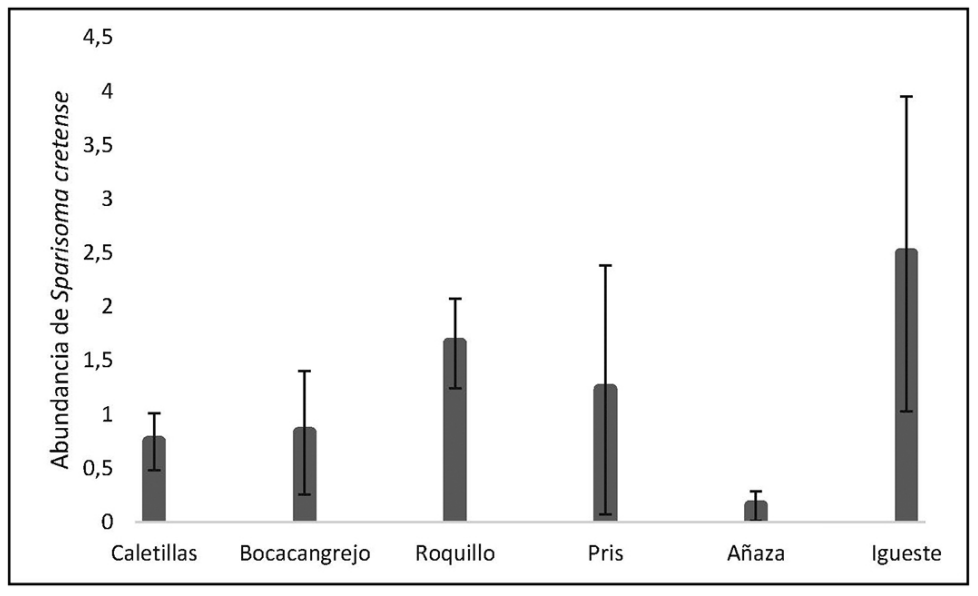

Fig. 4. Media de los individuos de talla mediana de S. cretense observados por tiempo muestreado en los distintos puntos de estudio. 